九州大学学術情報リポジトリ

Kyushu University Institutional Repository

\title{
A contribution to a notation system of the confounded factorial experiments
}

Kitagawa, Toshio

Kyushu University

Mitome, Michiwo

Kyushu Agricultural Experiment Station

https://doi.org/10.5109/12963

出版情報: 統計数理研究. 6 (1/2)，pp.1-10，1955-12. Research Association of Statistical Sciences

バージョン :

権利関係 : 


\title{
A CONTRIBUTION TO A NOTATION SYSTEM OF THE CONFOUNDED FACTORIAL EXPERIMENTS
}

\author{
By \\ Tosio Kitagawa and Michiwo Mitome \\ (Kyushu University and Kyushu Agricultural Experiment Station)
}

§1. Introduction. At present we have two different approaches for the study of the mathematical theory of factorial designs; one is the application of the theory of finite abelian groups proposed by Sir Ronald FIsHer $[2,3]$, the other is the application of finite geometry developed by R. C. BoSE [1] and his colleagues. Both FisheR's and BOSE's papers deal specifically the problems arising in the factorial arrangements. Our attention toward studying similar problems was started independently of their studies about 1948, when the only books referring to the design of experiments were Sir Ronald Fisher's [1] The Design of Experiments and F. YATEs's [1] Design and Analysis of Factorial Experiments. While we were making preparation for publishing of a book of Kitagawa, T. and Mitome, M. [2] entitled "Tables for the Design of Factorial Experiments" which embodies our results since 1948, H. B. MANN's [1] Analysis and Design of Experiments, W. G. Cochran and G. M. Cox's [1] Experimental Designs, and O. KemPTHORNE's [2] The Design and Analysis of Experiments, and others successively published. Unfortunately, we had already finished the essential part of our work before we could refer to their recent work. Here, we should like to point out that in our studies on the factorial design of experiments we used to take up three principles for the means of acquiring informations, namely, confounding, quasi-factor, and balancing in a broad sense.

The purpose of this paper is twofold: (i) to explain new notation systems which have been proposed by us elsewhere* and to review the confounded factorial designs in their use; (ii) to make a clear distinction between the types and the patterns of the confounded arrangements based on our notations and to enumerate them.

$\S 2$. The $\boldsymbol{p}^{n}$ factorial designs. When we have $n$ factors all at $p$ levels (where let $p$ be a prime number for convenience of illustration) and all independent, possible factorial combinations of which there will be $p^{n}$, and they may be represented as

$$
a^{\alpha} b^{\beta} c^{\gamma} \cdots n^{\nu} \quad(\alpha, \beta, \gamma, \cdots, \nu=0,1,2, \cdots, p-1) .
$$

\footnotetext{
* They were firstly communicated by the present authors at the Branch-Meeting of Japanese Math. Soc. held at Kumamoto University on Feb. 24, 1951. See also Kitagawa and Mitome $[1,2]$.
} 
All symbols of $p^{n}$ factorial or treatment combinations are said to form a finite abelian group of order $p^{n}$ and type $(p, p, \cdots, p)$ according to the commutative multiplication and ordinary algebraic rule with the condition

$$
a^{p}=b^{p}=c^{p}=\cdots=1 \text {. }
$$

At the same time, the symbols of $\left(p^{n}-1\right)$ independent comparisons or contrasts among $p^{n}$ factorial combinations, namely, $\boldsymbol{n}$ main effects of the factors, ${ }_{n} C_{k}$ ( $\left.2 \leqq k \leqq n\right) k$-factor interactions, and the identity 1 form the socalled contrast groups which are simply isomorphic to the treatment groups mentioned above. Now, let $\&$ be a treatment group of order $p^{n}$ or a prime power group modulo $p$. If $\mathfrak{F}$ is any subgroup of index $\nu$ under the group (S), then it can be decomposed in the form,

$$
\text { S }=\mathfrak{S}+\tilde{\mathfrak{C}} P_{1}+\mathfrak{S} P_{2}+\cdots+\mathfrak{\mathscr { C }} P_{\nu-1} .
$$

In this representation, $\tilde{\mathfrak{C}} P_{\lambda}(i=1,2, \cdots, \nu-1)$ stands for all the products formed by multiplying every element of $\tilde{\mathfrak{V}}$ into $\mathfrak{\mathcal { V }} P_{\lambda}$. The set of elements represented by $\mathfrak{\mathcal { C }} P_{\lambda}$ is called coset of $(\mathcal{S})$ as regards $\mathfrak{\mathcal { T }}$ which may conveniently be called the intrablock subgroup. Thus, the block that embodies intrablock subgroup may be termed the principal block. The compositions of the blocks of confounded arrangement may be obtained by two steps, firstly, by constructing the principal block, secondly, by choosing appropriate multipliers $P_{1}, P_{2}, \cdots, P_{\nu-1}$ for forming of cosets. Utilizing the orthogonality between the contrast or confounded subgroup and the intrablock subgroup, the principal block may be easily constructed. Two elements $A^{\alpha} B^{\beta} C^{\gamma} \cdots$ and $a^{\alpha^{\prime}} b^{\beta^{\prime}} c^{\gamma^{\prime}}$ $\cdots$ may be said to be orthogonal if

$$
\alpha \alpha^{\prime}+\beta \beta^{\prime}+\gamma \gamma^{\prime}+\cdots \equiv 0 \quad(\operatorname{riod} . p) .
$$

From the view point of the field experiments the procedure of confounding or the allocation of one replicate of treatment combinations to several blocks implies such allocation of all $p^{n}$ treatment combinations into $p^{s}(s<n)$ blocks of $p^{n-s}$ plots as the comparisons between these blocks become particular sets of $\left(p^{s}-1\right)$ independent comparisons. Viewed in the light of the group theory, it means that specified contrast subgroup consists of $p^{s}(1<s<n)$ elements is chosen from the contrast group of order $p^{n}$. The total number of subgroups of order $p^{s}$ in an abelian group of order $p^{n}$ is equal to

$$
N(\boldsymbol{n}, \boldsymbol{s})=\frac{\left(p^{n}-1\right)\left(p^{n-1}-1\right) \cdots\left(p^{n-s+1}-1\right)}{\left(p^{\varepsilon}-1\right)\left(p^{s-1}-1\right) \cdots(p-1)},
$$

where $N(n, s)$ stands for the total number of possible contrast subgroups. They are equivalent to whole patterns of confounding for a $p^{n}$ experiment in blocks of $p^{n-s}$. Clearly, if all $N(n, s)$ patterns of confounding are used in the experiments, the whole arrangements must be balanced, since each constrast is confounded with equal frequency over all replications. 
In many cases, however, it may be desirable from factorial principle to design the balanced confounded arrangements applying with the minimum number of patterns belong to the same type of arrangement. When the confounded subgroups or patterns are converted to each other by the interchange of the letters of denoting factors, it may be called that they are belong to the same type of confounded arrangement. When $p=2$, for example, it follows that, if one consists of $1, A, B C D, A B C D$, and the other consists of $1, B, A C D, A B C D$, then both patterns belong to the same type of arrangement since they are transposed to each other by substitution $(A \leftrightarrow B)$.

In order to elucidate the construction of confounded arrangement, and to distinguish the types from the patterns of the arrangement, we have been introduced a notation system, $p^{n}\left(p^{n-s}\right)[* * \cdots *]\left(i^{\circ}\right)$, where $p^{n}\left(p^{n-s}\right)$ denotes the subdivision of $p^{n}$ treatment combinations into $p^{s}$ blocks the size of $\left(p^{n-s}\right),[* * \cdots *]$ denotes the order of the confounded contrasts, and $\left(i^{\circ}\right)$ stands for the patterns listed in Kitagawa and Mitome [2]. For example, $2^{5}(8)$ [532] denotes a type of confounding for a $2^{5}$ experiment in 4 blocks each of $8\left(=2^{5-2}\right)$ whose confounded contrasts are three interactions, i.e., a 5-factor, a 3-factor, and a 2-factor interactions; $2^{\circ}(8)\left[4.3_{2}\right]\left(1^{\circ}\right)$ denotes a pattern of confounding whose confounded contrasts are $A B C, A D E$, and $B C D E$.

Now we shall give further examples in the following.

Case of 2 levels: $2^{4}(4)[4.31]\left(1^{\circ}\right) \sim\left(4^{\circ}\right), 2^{4}(4)\left[4.2_{2}\right]\left(1^{\circ}\right) \sim\left(3^{\circ}\right), 2^{4}(4)\left[3_{2} 2\right]$ $\left(1^{\circ}\right) \sim\left(6^{\circ}\right), 2^{4}(4)\left[\begin{array}{lll}3 & 2 & 1\end{array}\right]\left(1^{\circ}\right) \sim\left(12^{\circ}\right), 2^{4}(4)\left[2_{3}\right]\left(1^{\circ}\right) \sim\left(4^{\circ}\right), 2^{4}(4)\left[21_{2}\right]\left(1^{\circ}\right) \sim\left(6^{\circ}\right)$, these amount to in all $\left(2^{4}-1\right)\left(2^{3}-1\right) /\left(2^{2}-1\right)(2-1)=35$ patterns.

Case of 3 levels: $3^{3}(9)[3]\left(1^{\circ}\right) \sim\left(4^{\circ}\right)$, which represent the patterns given by Yates [1], i.e., $A B C(W), A B C(X), A B C(Y)$, and $A B C(Z)$, respectively.

Case of 4 levels: $4^{3}(16)\left[3_{3}\right]\left(1^{\circ}\right) \sim\left(9^{\circ}\right), 4^{3}(8)\left[3_{4} 2_{3}\right]$.

Case of 5 levels: $5^{3}(25)[3]\left(1^{\circ}\right) \sim\left(16^{\circ}\right), 5^{3}(25)[2]\left(1^{\circ}\right) \sim\left(12^{\circ}\right), 5^{3}(25)[1]$ $\left(1^{\circ}\right) \sim\left(3^{\circ}\right)$, the number of patterns is $\left(5^{3}-1\right) /(5-1)=31 ; 5^{3}(5)\left[3_{4} 21\right]$ $\left(1^{\circ}\right) \sim\left(12^{\circ}\right), 5^{3}(5)\left[3_{3} 2_{3}\right]\left(1^{\circ}\right) \sim\left(8^{\circ}\right), 5^{3}(5)\left[2_{4} 1_{2}\right]\left(1^{\circ}\right) \sim\left(3^{\circ}\right)$, in total 23 patterns make well-balanced arrangements in practice, instead of using $\left(5^{3}-1\right) \times$ $\left(5^{2}-1\right) /\left(5^{2}-1\right)(5-1)=31$ patterns.

Case of 7 levels: $7^{3}(4.9)[3]\left(1^{\circ}\right) \sim\left(36^{\circ}\right), 7^{3}(49)[2]\left(1^{\circ}\right) \sim\left(18^{\circ}\right), 7^{3}(4.9)[1]$ $\left(1^{\circ}\right) \sim\left(3^{\circ}\right)$, in all $\left(7^{3}-1\right) /(7-1)=57$ patterns; $7^{3}(7)\left[3_{6} 21\right]\left(1^{\circ}\right) \sim\left(18^{\circ}\right)$, $7^{3}(7)\left[3_{5} 2_{3}\right]\left(1^{\circ}\right) \sim\left(12^{\circ}\right), 7^{3}(7)\left[\begin{array}{ll}2_{6} & 1_{2}\end{array}\right]\left(1^{\circ}\right) \sim\left(3^{\circ}\right)$, although these 3 types also enumerate 57 patterns in all, using the patterns shown here every type may be attained the balancing, respectively (KITAGAwA and Mitome [2]).

§. The $\boldsymbol{p}^{m} \times \boldsymbol{q}^{n}$ factorial designs. In this section we shall consider the experiments in which some factors have different levels, namely, there are $m$ factors each at $p$ levels and $n$ factors each at $q$ levels $(p \neq q)$. We may deal them as the $p^{m} \times q^{n}$ type of factorial experiments, they may also be called the asymmetrical factorial designs or briefly mixed systems, while 
the $p^{m}$ designs are called the symmetrical factorial designs or pure systems, as described in the last section.

In order to design a confounded arrangement of a mixed system, e.g., $p^{m} \times q$ type, we usually take a well-known confounding pattern in $p^{m}$ type, i. e., $p^{m}()[\quad]\left(i^{\circ}\right)$, which may be combined with a factor having $q$ levels. Then, the constituent patterns $p^{m}(\quad)[\quad]\left(i^{\circ}\right) \times q_{s}(s=0,1,2, \cdots, q-1)$ will result. Rearranging the constituent patterns taking account of requirment for the balancing and the orthogonal conditions of the arrangement with available replicates, we may obtain a partial confounded arrangement of mixed system. Therefore, the confounding of mixed system is somewhat limited by those of fundamental pure system. The orthogonal conditions, which are especially important properties to mixed system, may be given by the primciples (i) (iv) mentioned below.

(i) Due to the orthogonal partition in Latin square.

Examples

$$
\begin{array}{lll}
\beta \alpha \alpha & 2^{2}(2)[*] \times 3, & 2^{3}(4)[*] \times 3, \\
\alpha \beta \alpha & 2^{4}(8)[*] \times 3, & 4.2(4)[2] \times 3 . \\
\alpha \alpha \beta & & \\
(2,1) &
\end{array}
$$

(ii) Due to the orthogonal set of Latin squares.

\section{Examples}

$$
\begin{array}{llll}
\beta \gamma & \delta & 2^{3}(2)[* * *] \times 3, & 2^{4}(4)[* * *] \times 3, \\
\gamma & \delta & 4^{2}(4)\left[2_{3}\right] \times 3 . & \\
\delta & \beta \gamma & & \\
\alpha & \beta \gamma \delta & 2^{2}(1)\left[21_{2}\right] \times 4, & 2^{3}(2)[* * *] \times 4 . \\
\beta & \alpha \delta \gamma & & \\
\gamma & \delta & \alpha &
\end{array}
$$

(iii) Due to the repetition of Latin squares.

\section{Examples}

$$
\begin{aligned}
& \begin{array}{lll}
\alpha \beta & 3^{2}(3)[*] \times 2, & 3^{3}(9)[3] \times 2,
\end{array} \\
& \beta \alpha \quad 3^{3}(3)\left[32_{2}\right] \times 2, \quad 4{ }^{2}(4)\left[2_{3}\right] \times 2 \text {, } \\
& 4 .^{2}(8)[2] \times 2, \quad 3^{3}(3)[2] \times 2^{2}(2)[2] .
\end{aligned}
$$

(iv) Due to the orthogonal partition in $2^{n}(n \geqq 2)$.

\section{Examples}

$$
\begin{array}{lllll}
\alpha & \alpha & \alpha & 2^{2}(2)[*] \times 4, & 2^{3}(4)[*] \times 4 . \\
\alpha & \beta & \beta \\
\beta & \beta & \alpha
\end{array} \quad \text {. }
$$


\$4. Fractional replication in factorial designs. The principles of fractional replication, which have been laid down mainly by FINNEY $[1,2]$ and KEMPTHORNE $[1,2]$, may be regarded as a further development of theoretical formulation of the confounding principle. 'The crucial part of specification of the confounded factorial design is clearly the choice of a set of confounded contrasts. They are known as the defining contrasts or confounding. Once the defining contrasts are chosen, we can specify the structure of confounded arrangement. Now, let (5) be an abelian group of order $p^{n}$, where $p$ is a prime number, $n$ is any positive integer, and type $(p, p, \cdots, p)$ then, the confounding with $p^{k}(k=1,2, \cdots, n-1)$ blocks of $p^{n-k}$ plots corresponds to the decomposition

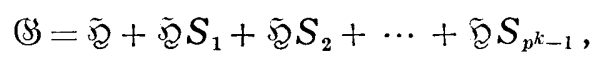

where $\mathfrak{T}$ is a subgroup of $\mathscr{G}$ as shown in $\S 2$. In such representation, what is called the fractional replication is the experiment testing on the subgroup $\mathfrak{\mathcal { S }}$ of order $p^{n-k}$, or any one coset $\tilde{\mathfrak{T}} S_{\nu}\left(\nu=1,2, \cdots, p^{k}-1\right)$ instead of using the whole group ( $S$ of order $p^{n}$. It is also properly called the $p^{n}-1 / p^{k}$ replicate design without confounding. When the subgroup $\mathfrak{S}$ is further decomposed into the cosets as regard a certain subgroup $\tilde{\mathfrak{S}}^{\prime}$ in the form

$$
\mathfrak{S}=\mathfrak{S}^{\prime}+\tilde{\mathfrak{S}}^{\prime} S^{\prime}{ }_{1}+\tilde{\mathfrak{S}}^{\prime} S^{\prime}{ }_{2}+\cdots+\tilde{\mathfrak{V}}^{\prime} S^{\prime}{ }_{p^{r}-1} \text {, }
$$

where $\tilde{\mathfrak{D}}$ is divided into $p^{r}$ blocks of $p^{n-k-r}$ plots, the design is called the $p^{n}-1 / p^{k}$ replicate design with confounding.

From such theoretical point of view, we shall now consider the following notations for the fractional replication with confounding:

$$
\begin{aligned}
& 2^{6}(32)[6]-1 / 4 \cdot[\mathfrak{g}], \quad 2^{7}(64)[7]-1 / 8[\mathfrak{g}], \\
& 2^{8}(128)[8]-1 / 8[\mathfrak{Q}], \quad 2^{8}(64)\left[65_{2}\right]-1 / 4 .[\$], \\
& 2^{9}(128)\left[\begin{array}{ll}
6 & 5_{2}
\end{array}\right]-1 / 8[\mathfrak{g}], \quad 2^{\prime \prime \prime}(128)\left[76_{3} 5_{3}\right]-1 / 8[\mathfrak{g}] \text {, } \\
& 2^{11}(128)\left[87_{2} 6_{6} 5_{6}\right]-1 / 8[\mathfrak{g}], \quad 2^{\prime 2}(256)\left[127_{4} 6_{6} 5_{4}\right]-1 / 16[\mathfrak{S}] \text {. }
\end{aligned}
$$

\begin{tabular}{|c|c|c|c|c|}
\hline $\begin{array}{l}\text { Factorial } \\
\text { systems } \\
p^{n} \equiv @\end{array}$ & $\begin{array}{c}\text { Fraction } \\
p^{n}\left(1 / p^{k}\right) \equiv \mathfrak{S}\end{array}$ & $\begin{array}{c}\text { Confounding } \\
\text { schemes } \\
1 / p^{r}[\mathfrak{W}]\end{array}$ & Block size & \\
\hline $2^{\mathrm{t} 2}$ & $2^{12}\left(1 / 2^{4}\right)$ & $1 / 2^{\prime \prime}$ & 256 & Without conf. \\
\hline $2^{11}$ & $2^{11}\left(1 / 2^{3}\right)$ & $1 / 2^{1}$ & 128 & With conf. \\
\hline $2^{10}$ & $2^{10}\left(1 / 2^{2}\right)$ & $1 / 2^{2}$ & 64 & With conf. \\
\hline $2^{9}$ & $2^{9}\left(1 / 2^{1}\right)$ & $1 / 2^{3}$ & 32 & With conf. \\
\hline $2^{s}$ & $2^{s}\left(1 / 2^{\prime \prime}\right)$ & $1 / 2^{4}$ & 16 & Full rep. \\
\hline
\end{tabular}

These represent the types of defining confounding or alias subgroup and the confounding scheme for intrablock subgroup. It should be noted, in passing, that a group of lower-ordered fractional replication are provided in succession from a fixed high-ordered one as mentitioned below: 
Recently, Kitagawa [1] proposed a reformed notation such as $p^{n}\left(p^{n-k-r}\right)$ $[\gamma \quad \partial]-1 / p^{k}$ in place of $p^{n}\left(p^{n-k}\right)[\gamma]-1 / p^{r}[\mathfrak{S}]$, where $\gamma$ and $o$ represents the defining contrasts and the confounded contrasts respectively.

\$5. Factorial designs with split-plot confounding. In field experiments with unifactor or factorial system, there is a device of splitting the plots by which an additional factor or factorial combinations can be introduced into the experiments. These designs are known as split-plot designs. In such a case the basic plots within a replicate are called the whole-plots or main-plots, and the subdivided plots within main-plot are called the subplots.

In view of the structure of the experiment, there are two sets of factors, namely, one set being assigned to the main-plots and the other to the splitplots within each main-plot, thus, these two sets may be called the main-plot factors and the subplot factors, respectively.

Clearly the allocation of these two sets of factors is carried out independently each other to some extent, and the main-plots may be arranged either in randomized blocks or in Latin square as the fundamental arrangements. The confounding may or may not be used in such designs. When each main-plot is devided into the subplots whose number is insufficient for the allocation of subplot treatment, and then, all treatments of the additional factors are not applied on the sections of any one main-plot, consequently, certain contrasts between subplot treatments are evaluated by main-plot difference. This is called the split-plot design with confounding. On the contrary, if each main-plot being devided into as many subplots as there are subplot treatments, it is called the split-plot design without confounding, for which there are no difficulties in the arrangement, since the problems depend only upon the splitting of main-plot as for the available subplots. In either cases, however, the main-plot factor effects being confounded with the main-plot differences, which should be regarded as the proper confounding to this design and they are likely to be of neglegible importance in practice.

It is desirable to develop a generalized split plot principle for further extension of the designs, because certain types of quasi-Latin squares and of other research branches are also expected to be utilized as the fundamental arrangements of the main-plot factors.

The symbolism representing the types of arrangements is the straightforward extension of those of ordinary factorial designs. They take commonly the symbol, $\alpha(\beta)[\gamma]$, where $\alpha$ represents the factorial system, ( $\beta$ ) represents the block size or its constitution, and [r] represents the order of confounded comparisons which being confounded among blocks, but, in Latin square they are separated by a semicolon for indicating of distinction between the confounding among columns and those among rows. When 
$\alpha=6 \cdot 2^{3}$ and $\beta=(6 \times 2) \cdot 4$, for example, it means that there is a split-plot design which has a main-plot factor at 6 levels with together 3 subplot factors each at 2 levels, and that the factorial combinations are arranged in a block of $6 \times 2$ main-plots each having 4 subplots. The following examples may be available for using of the split-plot confounding in Latinsquare.

$$
\begin{array}{ll}
4 \cdot 2^{2}((4 \times 4) \cdot 2)[3 ; 3], & 4 \cdot 2^{3}((4 \times 4) \cdot 2)[3 ; 3], \\
4 \cdot 2^{3}((4 \times 4) \cdot 4)[4 ; 4], & 4 \cdot 2^{3}((4 \times 4) \cdot 4)[3 ; 3,3, \\
4 \cdot \cdot 2^{3}((4 \times 4) \cdot 2)[3 ; 3], & 6 \cdot 2^{3}((6 \times 6) \cdot 2)[3 ; 3], \\
6 \cdot 2^{3}((6 \times 6) \cdot 4)[3 ; 4], & 6 \cdot 2^{3}((6 \times 6) \cdot 4)[4 ; 4], \\
6 \cdot 3^{3}((6 \times 6) \cdot 3)[3 ; 3] . &
\end{array}
$$

§. Factorial designs confounded in quasi-Latin squares. Let there be one replicate of treatment combinations which may be arranged $m$ groups each of $k$ treatments. If we take $k=m \boldsymbol{r}$, then a $k \times k$ I.atin square arrangement with $r$ replicates can be constructed so that some higher order interactions are confounded with the rows and others with the columns. Such square arrangements which have the advantages due to the principle of Latin square are generally called quasi-Latin squares, especially, they form ordinary Latin squares when $k=m$.

In these confounded arrangements, it may be possible to combine additional or subsidiary factors with the main factors forming a quasi-Latin square. The following are the examples which are classified according to the number of subsidiary factors, based on the principles outlined by YATES [1].

(1) Case of those without subsidiary factors. In view of the aim of experiment there are no proper differences between main and subsidiary factors.

Examples : $2^{3}(4 \times 4)[3 ; 2], \quad 2^{3}(4 \times 4)[3 ; 2]$,

$$
\begin{array}{lll}
2^{3}(4 \times 4)[32 ; 2], & 2^{3}(4 \times 4)[32 ; 2, & 2^{3}(4 \times 4)[2 ; 2], \\
2^{4}(8 \times 8)\left[4 ; 3_{4}\right], & 2^{6}(8 \times 8)\left[4 ; 3 ; 4 ; 3_{4}\right], & 3^{3}(9 \times 9)[3 ; 3] .
\end{array}
$$

A derivation of quasi-Latin squares from confounded factorial arrangements shown in $\S 2$ will be seen in the following illustrative examples:

$$
\begin{aligned}
& 2^{4}(4 . \times 4)\left[4.22_{2} ; 3_{2} 2\right]\left\{\begin{array}{l}
\text { Columns: } 2^{4}(4)[A B C D, A B, C D] \\
\text {. }
\end{array}\right. \\
& \text { \{ Rows: } \quad 2^{4}(4)[A B C, B C D, A D] \\
& 2^{5}(8 \times 8)\left[\begin{array}{lll}
4 & 3_{4} ; 4_{2} 3_{4}
\end{array}\right] \begin{cases}\text { Columns : } & 2^{5}(8)[A B D E, A C E, B C D] \\
& 2^{5}(8)[A B C E, A C D, B D E] \\
\text { Rows : } & 2^{5}(8)[B C D E, A B C, A D E] \\
& 2^{5}(8)[A C D E, A B D, B C E]\end{cases} \\
& 3^{3}(9 \times 9)\left[3_{3} ; 3_{3}\right] \\
& \text { Columns: } 3^{3}(9)[A B C], 3^{3}(9)\left[A B C^{2}\right], 3^{3}(9)[A B C] \\
& \text { Rows: } \quad 3^{3}(9)\left[A B^{2} C^{2}\right], 3^{3}(9)\left[A B^{2} C\right], 3^{3}(9)\left[A B^{2} C\right] \\
& 3^{4}(9 \times 9)\left[3_{4} ; 3_{4}\right] \\
& \text { Columns: } 3^{4}(9)\left[A B C^{2}, A B^{2} D, A C D^{2}, B C D\right] \\
& \text { l Rows: } \quad 3^{4}(9)\left[A B^{2} C^{2}, A B D^{2}, A C D, B C^{2} D\right]
\end{aligned}
$$


$(2)_{1}$ Case of the non-plaid square with one subsidiary factor. Although one of the factors has only subsidiary meaning as to the experiment, it is handled as same as the main factors.

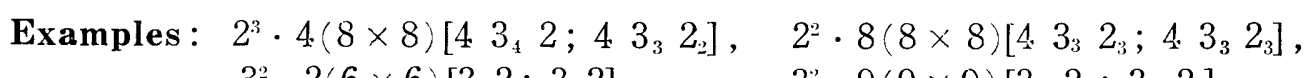
$3^{2} \cdot 2(6 \times 6)[32 ; 32], \quad 3^{2} \cdot 9(9 \times 9)\left[3_{2} 2_{2} ; 3_{2} 2_{2}\right]$.

$(2)_{2}$ Case of the half-plaid square with one subsidiary factor. This square has a plaid pattern in one direction of rows or columns as for the level of subsidiary factor. Consequently, the main effect of subsidiary factor is confounded with rows (or columns), in this respect it may be considered that this design connects with an idea of split-plot designs.

Examples: $2^{2} \cdot(4 \times 4)[3 ; 1], \quad 2 \cdot 4 \cdot(4 \times 4)[32 ; 1]$, $\begin{array}{ll}2^{3} \cdot 2(8 \times 8)[4 ; 1], & 2^{4} \cdot 2(8 \times 8)[4.3 ; 541], \\ 2^{3} \cdot 4(8 \times 8)[3 ; 1], & 3 \times 2 \cdot 2(6 \times 6)[32 ; 1], \\ 3 \times 2 \cdot 3(6 \times 6)[-; 21] . & \end{array}$

$(3)_{1}$ Case of the half-plaid square with two subsidiary factors. This design is easily constructed as a complex of case $(2)_{1}$ and case $(2)_{2}$.

Example: $3^{2} \cdot 3 \cdot 3(9 \times 9)\left[3_{4} ; 4_{2} 31\right]$.

$(3)_{2}$ Case of the plaid square with two subsidiary factors. This design is a straight-forward development of case $(2)_{2}$. One of two subsidiary factors goes to the rows and the other goes to the columns of the square, then a Scotch plaid pattern will result.

Examples: $2^{3} \cdot 2 \cdot 2(8 \times 8)[54.1 ; 4.31]$,

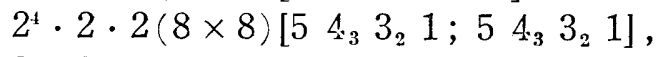
$3^{2} \cdot 3 \cdot 3(9 \times 9)\left[4_{2} 31 ; 4 ; 231\right]$.

§ 7. Lattice designs. We herein propose to consider the problems of testing a large number of varieties or treatments, so for the correspondence between the varieties and the treatment combinations of the factorial system. In these designs both the principles of subdivision of experimental materials and of confounding are ingeniously applied to their arrangements in order to eliminate heterogeneity of materials to a greater extent than that may be expected in ordinary designs. We should like to emphasize these two aspects of the lattice designs. The first point of view regards the subdividing of one replicate of varieties or treatments tested, e.g., $v=k \times b$, into $b$ (or $k$ ) blocks of $k$ (or $b$ ) varieties each, that is to say the lattice designs are the incomplete block designs. The second point of view regards the partial confounding with some comparisons among varieties, where it is desired to retain as much information as possible and to make the balanced information concerning all comparisons. It is also desired that any variety difference is estimated with approximately the equal variance as well as reasonably small variance in the analysis of variance procedure. 
We now suppose two factors $X$ and $Y$ at $k, b$ levels each, with which $k \times b$ lattice having $k b$ points will result. If $k b$ varieties are superimposed on these $k b$ lattice points, we have a one-to-one correspondence between the factorial combinations $k \times b$ and the $k b$ varieties. Thus, the general properties of factorial arrangement are come out on a mere aggregate of varieties. This may be called the formal establishment of quasifactors.

We have described above the fundamental type of the lattice designs which originated in YATES[1]. The name "lattice" is a remainder of a step of the development of factorial designs, though at present we using it generally as it expresses the originator's idea under the name of "lattice." However, in view of the factorial principle, they should be embodied in the name of "quasifactorial designs."

Now we shall consider the structure of the 3-dimensional lattice with 3 groups from the viewpoint of factorial arrangement. For example, let us suppose the formal correspondence between the arrangement of $125\left(=5^{3}\right)$ varieties into $5^{2}$ incomplete blocks of 5 plots and the confounding of $5^{3}(5)$ $\left[\left(5^{2}-1\right) d . f.\right]$ type which has $\left(5^{3}-1\right) /(5-1)=5^{2}+5+1$ different possible patterns. Among these the following 3 patterns give a solution used for usual practice, namely,

$$
\begin{array}{ll}
X \text {-group : } & 5^{3}(5)\left[2_{4} 1_{2}\right]\left(3^{\circ}\right) ; B C, B C^{2}, B C^{3}, B C^{4}, B, C \\
Y \text {-group : } & 5^{3}(5)\left[\begin{array}{ll}
2_{4} & 1_{2}
\end{array}\right]\left(2^{\circ}\right) ; A C, A C^{2}, A C^{3}, A C^{4}, A, C \\
Z \text {-group : } & 5^{3}(5)\left[\begin{array}{ll}
2_{4} & 1_{2}
\end{array}\right]\left(1^{\circ}\right) ; A B, A B^{2}, A B^{3}, A B^{4}, A, B
\end{array}
$$

Examples of $4 \times 4$ lattice squares given in Kitagawa and Mitome [2] have the following quasifactorial construction.

\begin{tabular}{r|c|c|c|c}
\hline \multirow{2}{*}{ Square No. } & \multicolumn{2}{|c|}{ Rows } & \multicolumn{2}{c}{ Columns } \\
\cline { 2 - 5 } & $\begin{array}{l}\text { Confounding } \\
\text { patterns }\end{array}$ & $\begin{array}{l}\text { Confounded } \\
\text { comparisons* }\end{array}$ & $\begin{array}{l}\text { Confounding: } \\
\text { patterns }\end{array}$ & $\begin{array}{l}\text { Confounded } \\
\text { comparisons* }\end{array}$ \\
\hline$\left(1^{\circ}\right) \approx\left(2^{\circ}\right)$ & $2^{4}(4)\left[21_{2}\right]\left(1^{\circ}\right)$ & $A, B$ & $2^{4}(4)\left[21_{2}\right]\left(6^{\circ}\right)$ & $C, D$ \\
$\left(3^{\circ}\right)$ & $2^{4}(4)\left[42_{2}\right]\left(2^{\circ}\right)$ & $A C, B D$ & $2^{4}(4)\left[3_{2} 2\right]\left(3^{\circ}\right)$ & $A D, A B C$ \\
$\left(4^{\circ}\right)$ & $2^{4}(4)\left[3_{2} 2\right]\left(3^{\circ}\right)$ & $A D, A B C$ & $2^{4}(4)\left[3_{2} 2\right]\left(4^{\circ}\right)$ & $B C, A B D$ \\
$\left(5^{\circ}\right)$ & $2^{4}(4)\left[3_{2} 2\right]\left(4^{\circ}\right)$ & $B C, A B D$ & $2^{\prime}(4)[42.2]\left(2^{\circ}\right)$ & $A C, B D$
\end{tabular}

* Only generators are shown.

\section{References}

Bose, R. C. [1] Mathematical theory of the symmetrical factorial designs. Sankhyā, 8 (1947), $107-166$.

Bose, R. C., and Kishen, K. [1] On the problem of confounding in the general symmetrical factorial design. Sankhyā, 5 (1940), 21-36.

Brownlee, K. A. [1] Industrial experimentation. Chemical Publishing Co., Inc., Brooklyn, N. Y., 4th Amer. ed., 1952.

Davies, O. L. (ed.) [1] The design and analysis of industrial experiments, Oliver \& Boyd, Edinburgh, 1954. 
Cochran, W. G., and Cox, G. M. [1] Experimental designs. John Wiley \& Sons, Inc., New York, 1950.

Federer, W. T. [1] Experimental design-Theory and Application. Macmillan Co., N.Y. 1955.

Finnex, D. J. [1] The fractional replication of factorial arrangements. Ann. Eugen., 12 (1945), $291-301$.

Finney, D. J. [2] Recent developments in the design of field experiments. III. Fractional replication. Jour. Agr. Sci., 36 (1946), 184-191.

Fisher, R. A. [1] The design of experiments. Oliver \& Boyd, Edinburgh, 1935.

Fister, R. A. [2] The theory of confounding in factorial experiments in relation to the theory of groups. Ann. Eugen., 11 (1942), 341-353.

Fisher, R. A. [3] A system of confounding for factors with more than two alternatives, giving completely orthogonal cubes and higher powers. Ann. Eugen., 12 (1945) 283-290.

KeMpthorne, $\mathrm{O}$. [1] A simple approach to confounding and fractional replication in factorial experiments. Biometrika, 34 (1947), 255-272.

Kempthorne, O. [2] The design and analysis of experiments. John Wiley \& Sons, Inc., New York, 1952.

KitaGaWA, T. [1] Lectures on the design of experiments, II. (in Japanese), Baifukan Co., Ltd., Tokyo, (in press).

Kitagawa, T. and Mitome, M. [1] Report on the accomplishment of "Tables for the design of factorial experiments." in Advances in probability theory and stochastics. Iwanami Pub. Co, Tokyo, 1953. (in Japanese)

Kitagawa, T. and Mitome, M. [2] Tables for the design of factorial experiments. Baifukan Co., Ltd., Tokyo, 1953. (Introductory chapters and commentary of tables are in Japanese, but the tables themselves are elucidated entirely in English.)

LI, J. C. R. [1] Design and statistical analysis of some confounded factorial experiments. Iowa Agr. Exp. Stat. Res. Bull., 333 (1944), 452-492.

MaNN, H. B. [1] Analysis and design of experiments. Dover Publications, Inc., New York, 1949.

NAIR, K. R., and RAO, C. R. [1] Confounding in asymmetrical factorial experiments. Jour. Roy. Stat. Soc., B, 10 (1948), 109-131.

Quenoujlle, M. H. [1] The design and analysis of experiment. Charles Griffin \& Co. Ltd., London, 1953.

RAo, C. R. [1] Confounded factorial designs in quasi-Latin squares. Sankhyā, 7 (1946), 295-304.

Rao, C. R. [2] A simplified approach to factorial experiments and the punched card technique in the construction and analysis of designs. Bull. Internatinal Stat. Inst. Vol. 33 (1951), Part 2, 1-28.

YATES, F. [1] The design and analysis of factorial experiments. Imp. Bur. Soil Sci., Harpenden, England, 1937. 